\title{
VE-PTP phosphatase: a new target for diabetic kidney disease
}

\section{$c 6$}

We are keen

to determine

if these drugs

might 'reset'

the endothelial

phenotype

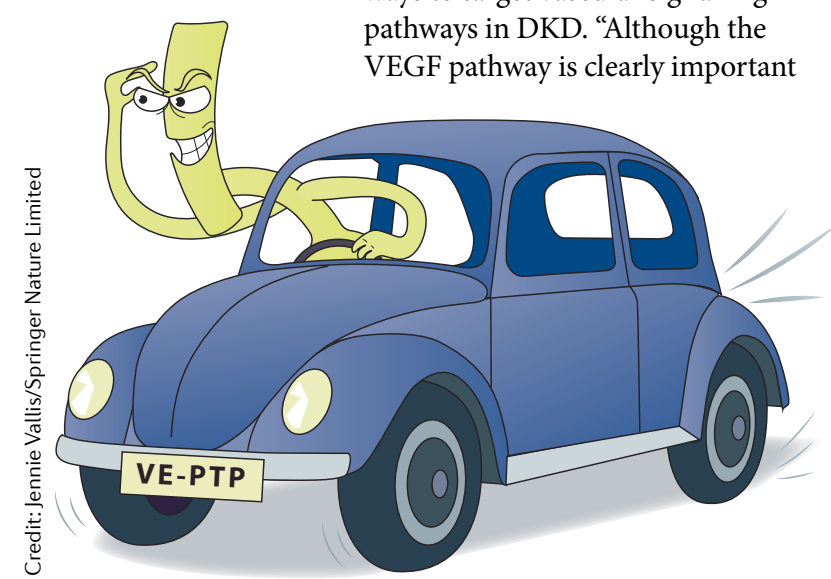

in vascular disease, it is tough to target in kidney disease due to the exquisite sensitivity of the glomerular capillaries to this growth factor. Hence we turned to another vascular tyrosine kinase pathway, the angiopoietin-TIE2 pathway." Previous studies by Quaggin and others had shown that activation of TIE2 promotes vascular stability whereas loss of angiopoietin 1 or TIE2 induces kidney failure in diabetic animals. "While others have focused on the levels and activity of the growth factor ligands, we looked at the possibility of targeting a negative regulator of the kinase - VE-PTP," says Quaggin.

The finding that VE-PTP expression is upregulated in the vasculature of diabetic mice led the researchers to develop new genetic mouse models to test whether activating TIE2 by inhibiting VE-PTP could protect mice from DKD. As expected, postnatal deletion of VE-PTP in mice resulted in robust activation of TIE2. Non-diabetic VE-PTP-knockout mice had normal lifespan, but had lower blood pressure (BP) and higher glomerular filtration rate than wild-type mice as a result of increased endothelial nitric oxide synthase (eNOS) phosphorylation and cortical renal blood flow.

To study the effect of VE-PTP inhibition on the development of DKD, Quaggin and colleagues crossed inducible VE-PTP-knockout mice with diabetic Akita mice and administered a renin-expressing adeno-associated virus (AAV) to induce a hypertensive response. Despite similar increases in BP and blood glucose levels in diabetic VE-PTP-knockout and diabetic control mice both before and after administration of $\mathrm{AAV}$, diabetichypertensive VE-PTP-knockout mice were protected from the decline in renal function observed in diabetichypertensive control mice. Diabetic-hypertensive VE-PTPknockout mice were also protected from glomerular histopathological damage. Further analyses showed that deletion of VE-PTP was associated with decreased expression of the transcription factor FOXO1 and its downstream profibrotic and pro-inflammatory targets. "We therefore propose that upregulation of VE-PTP in the vasculature of diabetic mice leads to a reduction of vasculoprotective pathways such as those regulated by eNOS, as well as the activation of harmful pathways such as those mediated by FOXO1," explains Quaggin. "Of note, a clinical trial of AKB-9778, which targets VE-PTP, in patients with diabetic macular oedema recently reported disappointing mid-term results in that there was no significant benefit of this drug for the disease; however, the trial showed that this agent was safe and, interestingly, a secondary outcome a reduction in proteinuria - was observed in treated patients."

Quaggin and colleagues are currently developing therapies to activate TIE2. "These drugs will be formulated in such a way as to target a number of different vascular diseases in the kidney," she says. "We are keen to determine if these drugs might 'reset' the endothelial phenotype so that acute, short-term administration might provide long-term benefit."

Susan J. Allison

ORIGINAL ARTICLE Carota, I. A. et al. Targetin VE-PTP phosphatase protects the kidney from diabetic injury.J. Exp. Med. https://doi.org/ 10.1084/jem.20180009 (2019) 